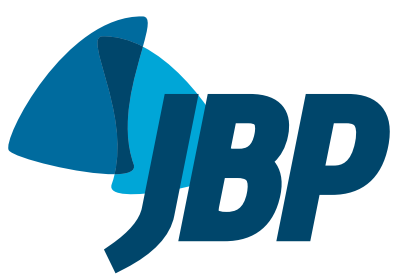

Multiple calcified nodules

Edson Marchiori ${ }^{1,2}$, Gláucia Zanetti ${ }^{2,3}$, Bruno Hochhegger ${ }^{4,5}$
A 22-year-old male patient previously diagnosed with osteosarcoma in the right leg, which was amputated in 2011, sought medical attention. The patient had multiple pulmonary nodules with calcifications (Figure 1). The final diagnosis was calcified metastases from osteosarcoma.

Multiple pulmonary nodules can have numerous etiologies. However, when these lesions have calcifications, the diagnostic possibilities are considerably reduced. Calcified metastases, amyloidosis, hyalinizing granulomas, epithelioid hemangioendothelioma, rheumatoid nodules, and multiple chondromas can be listed as the major causes of the pattern consisting of multiple nodules and/or masses of various sizes and with calcifications. The tumors that most commonly give rise to calcified metastases are sarcomas, especially osteosarcomas; synovial sarcomas; chondrosarcomas; and carcinomas, particularly mucinous or papillary adenocarcinomas.

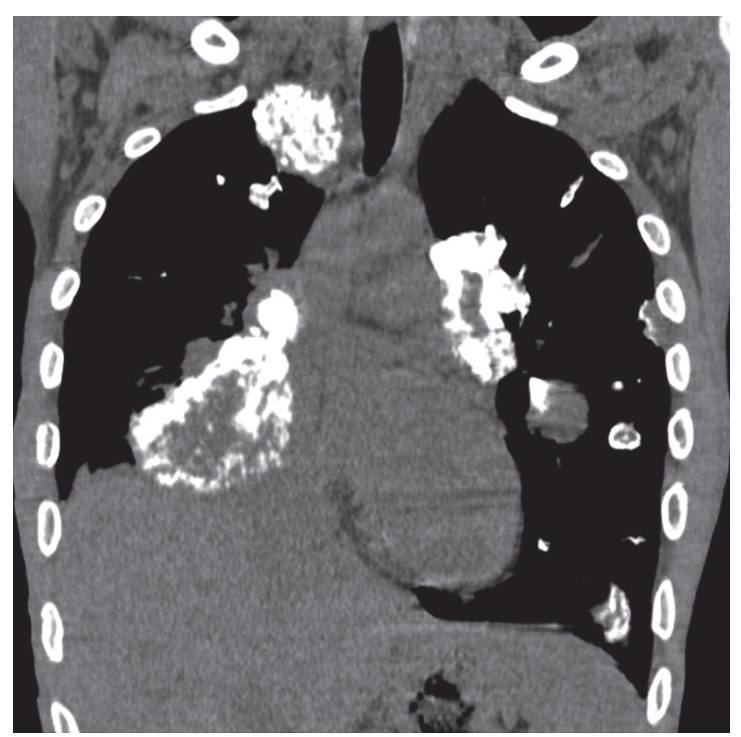

Figure 1. Coronal CT section (mediastinal window settings) showing multiple partially calcified nodules and masses, of various sizes, in both lungs.
Amyloidosis, in its nodular form, is more common in patients over 60 years of age, and these patients are usually asymptomatic. The nodules grow slowly, over years, without regression. The definitive diagnosis is made by histopathology, on the basis of the finding of deposition of amyloid, which stains with Congo red and shows apple-green birefringence in polarized light. Hyalinizing granulomas are rare fibrotic pulmonary lesions, usually associated with autoimmune phenomena mainly related to exposure to mycobacterial or fungal antigens. These lesions can remain stable or grow slowly for years. Epithelioid hemangioendothelioma is a rare multifocal pulmonary neoplasm of endothelial origin. It is considered a sarcoma of low aggressiveness. Although it does not usually exhibit growth, it eventually grows slowly and can lead to respiratory failure. In addition, it can develop calcification within 10-20 years of diagnosis. Necrobiotic nodules can develop in patients with pneumoconiosis associated with rheumatoid arthritis. The onset of these nodules characterizes Caplan's syndrome. Calcification in pulmonary chondromas is a common radiological finding. The association of these chondromas with gastrointestinal stromal tumors and extra-adrenal paragangliomas is known as the Carney triad. In all these diseases, patients can be asymptomatic or present with nonspecific chest symptoms. Therefore, the lesions are often incidental radiological findings. The association between clinical and radiological findings can significantly improve the accuracy of the diagnosis. However, in most cases, lung biopsy with immunohistochemical staining is required to establish the correct diagnosis. In the case of the patient in question, the history of osteosarcoma resection associated with the presence of calcified pulmonary nodules led to the final diagnosis of metastases from osteosarcoma. This diagnosis is facilitated, in the vast majority of cases, because the primary bone tumor is already known before the onset of metastases.

\section{RECOMMENDED READING}

1. Webb WR, Muller NL, Naidich DP, editors. High-resolution CT of the lung. 4th ed. Philadelphia: Lippincott Williams \& Wilkins; 2008. 